UMTRI-2005-32

\title{
INFLUENCE OF THE VISIBILITY OUT OF THE VEHICLE CABIN ON LANE-CHANGE CRASHES
}

\author{
Michael Sivak \\ Brandon Schoettle \\ Matthew P. Reed \\ Michael J. Flannagan
}

November 2005 
INFLUENCE OF THE VISIBILITY OUT OF THE VEHICLE CABIN ON LANE-CHANGE CRASHES

\author{
Michael Sivak \\ Brandon Schoettle \\ Matthew P. Reed \\ Michael J. Flannagan
}

The University of Michigan

Transportation Research Institute

Ann Arbor, Michigan 48109-2150

U.S.A.

Report No. UMTRI-2005-32

November 2005 
Technical Report Documentation Page

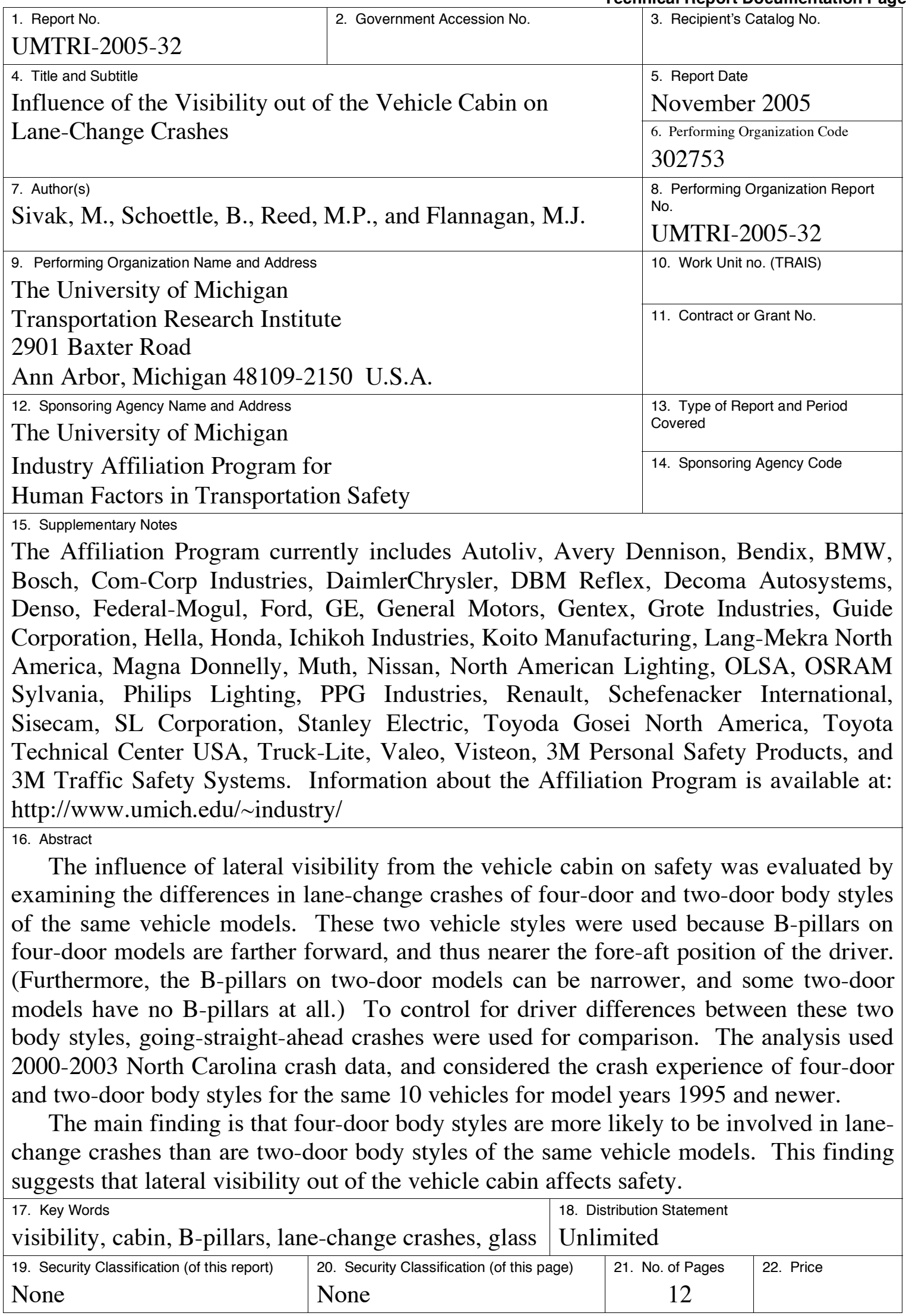




\section{Acknowledgment}

Appreciation is extended to the members of the University of Michigan Industry Affiliation Program for Human Factors in Transportation Safety for support of this research. The current members of the Program are:

\begin{tabular}{|c|c|}
\hline Autoliv & Lang-Mekra North America \\
\hline Avery Dennison & Magna Donnelly \\
\hline Bendix & Muth \\
\hline BMW & Nissan \\
\hline Bosch & North American Lighting \\
\hline Com-Corp Industries & OLSA \\
\hline DaimlerChrysler & OSRAM Sylvania \\
\hline DBM Reflex & Philips Lighting \\
\hline Decoma Autosystems & PPG Industries \\
\hline Denso & Renault \\
\hline Federal-Mogul & Schefenacker International \\
\hline Ford & Sisecam \\
\hline GE & SL Corporation \\
\hline General Motors & Stanley Electric \\
\hline Gentex & Toyoda Gosei North America \\
\hline Grote Industries & Toyota Technical Center, USA \\
\hline Guide Corporation & Truck-Lite \\
\hline Hella & Valeo \\
\hline Honda & Visteon \\
\hline Ichikoh Industries & 3M Personal Safety Products \\
\hline Koito Manufacturing & 3M Traffic Safety Systems \\
\hline
\end{tabular}




\section{Contents}

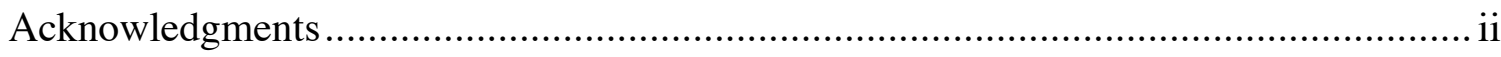

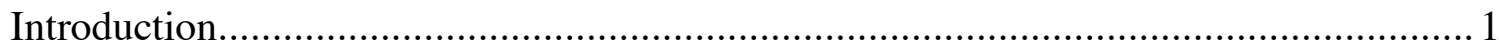

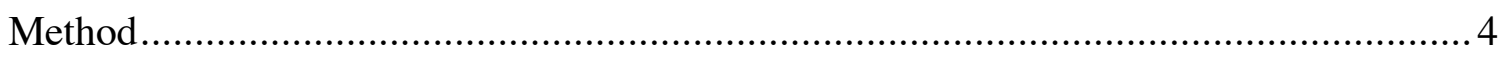

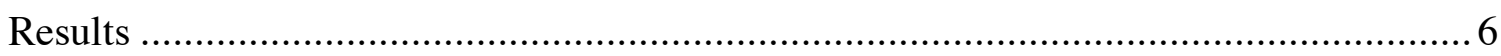

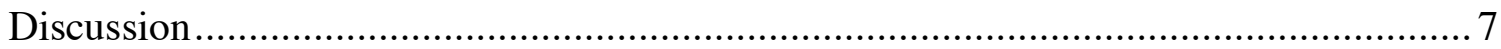

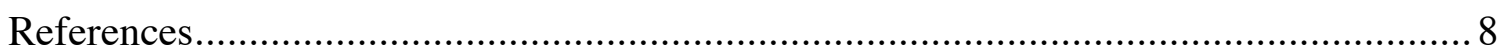




\section{Introduction}

\section{Background}

The importance of the extent of the transparent (glass) area of the vehicle shell is easy to ascertain for the extreme case: If there were no transparent areas, driving (as we know it) would not be possible. However, the incremental safety consequences of specific opaque areas in the direct field of drivers' vision have not yet been quantified.

This study was designed to provide some initial assessment of the potential safety importance of the location of B-pillars by examining lane-change crashes as a function of the body style (two-door vs. four-door). In general, in comparison to two-door models, the B-pillars on four-door models are farther forward and thus nearer the fore-aft position of the driver. (See Figure 1.) (Furthermore, the B-pillars on two-door models can be narrower, and some two-door models have no B-pillars at all.) Consequently, the lateral visibility should be better in two-door models than in four-door models, and this may result in two-door models having fewer lane-change crashes.

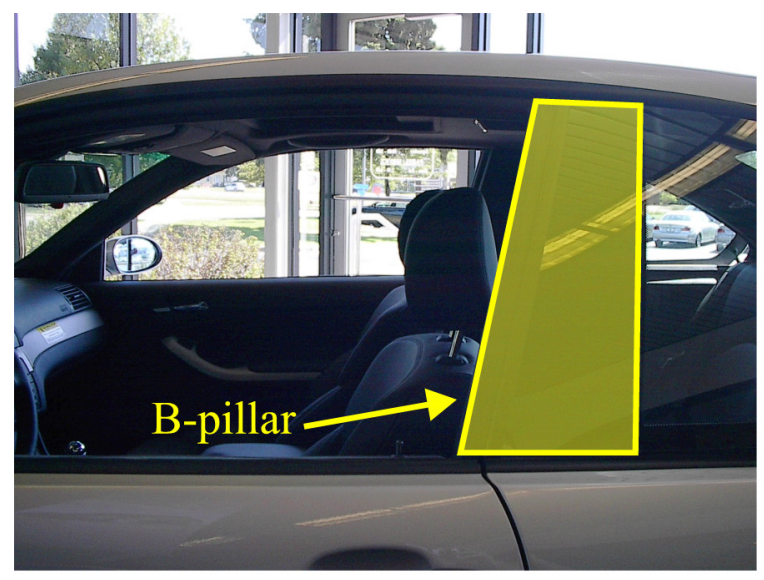

Two-door

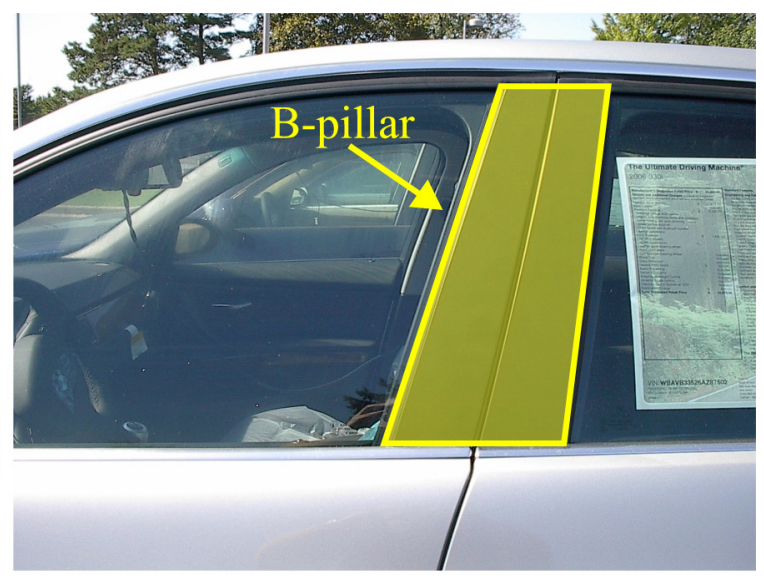

Four-door

Figure 1. An example of the location of the B-pillars (highlighted) for two-door (left) and four-door (right) body styles of the same vehicle model. 
However, a simple comparison of lane-change crashes of four-door and two-door models would be confounded by driver differences for the two body styles (e.g., age, gender, income, education, etc.). These driver differences are likely to influence the amount and type of driving exposure (e.g., ambient-illumination and road-type distributions), as well as driving style (e.g., risk-taking tendency, driving while fatigued or intoxicated). Therefore, we used the involvement of the two vehicle body styles in crashes that involve going straight ahead as a control because this type of crashes should not be affected by lateral visibility. Furthermore, the analysis included only those vehicle models that were available in both body styles.

\section{B-pillars and lateral visibility: An illustrative example}

To illustrate the lateral visibility differences between two-door and four-door models, the relevant interior and exterior geometry was measured for two-door and fourdoor body styles of the same vehicle model. Using a FARO Arm coordinate measurement machine, the outlines of the driver window, A-pillar, and B-pillar were recorded. The locations of the steering wheel and accelerator pedal were measured, and the seat H-point travel path was measured using the SAE J826-1995 manikin (SAE, 1995). The relationships among the steering wheel, pedals, and seat track were identical between the two vehicles within measurement error. Using the steering wheel location and seat-track adjustment range, the 95th-percentile SAE J941 eyellipse (SAE, 2002) was located within the vehicle cabin. Under the assumptions of the eyellipse, 95\% of drivers' eyes are predicted to lie on one side of any tangent to the eyellipse, and $74 \%$ of drivers' eyes are predicted to lie within the side-view or top-view perimeter of the eyellipse.

Figure 2 shows the eyellipse along with the door-window outlines from the two vehicles. The B-pillar in this four-door model is approximately $210 \mathrm{~mm}$ forward of its location in the corresponding two-door model on the horizontal plane passing through the eyellipse centroid. In this plane, the angle of a tangent to the back of the left-eye eyellipse passing tangent to the B-pillar forms an angle with a lateral axis of 45 degrees in the two-door model and 17 degrees in the four-door model. (A more complete consideration of lateral view in these vehicles would include consideration of head turn. However, the relative assessment of the two vehicles would remain essentially unchanged.) 


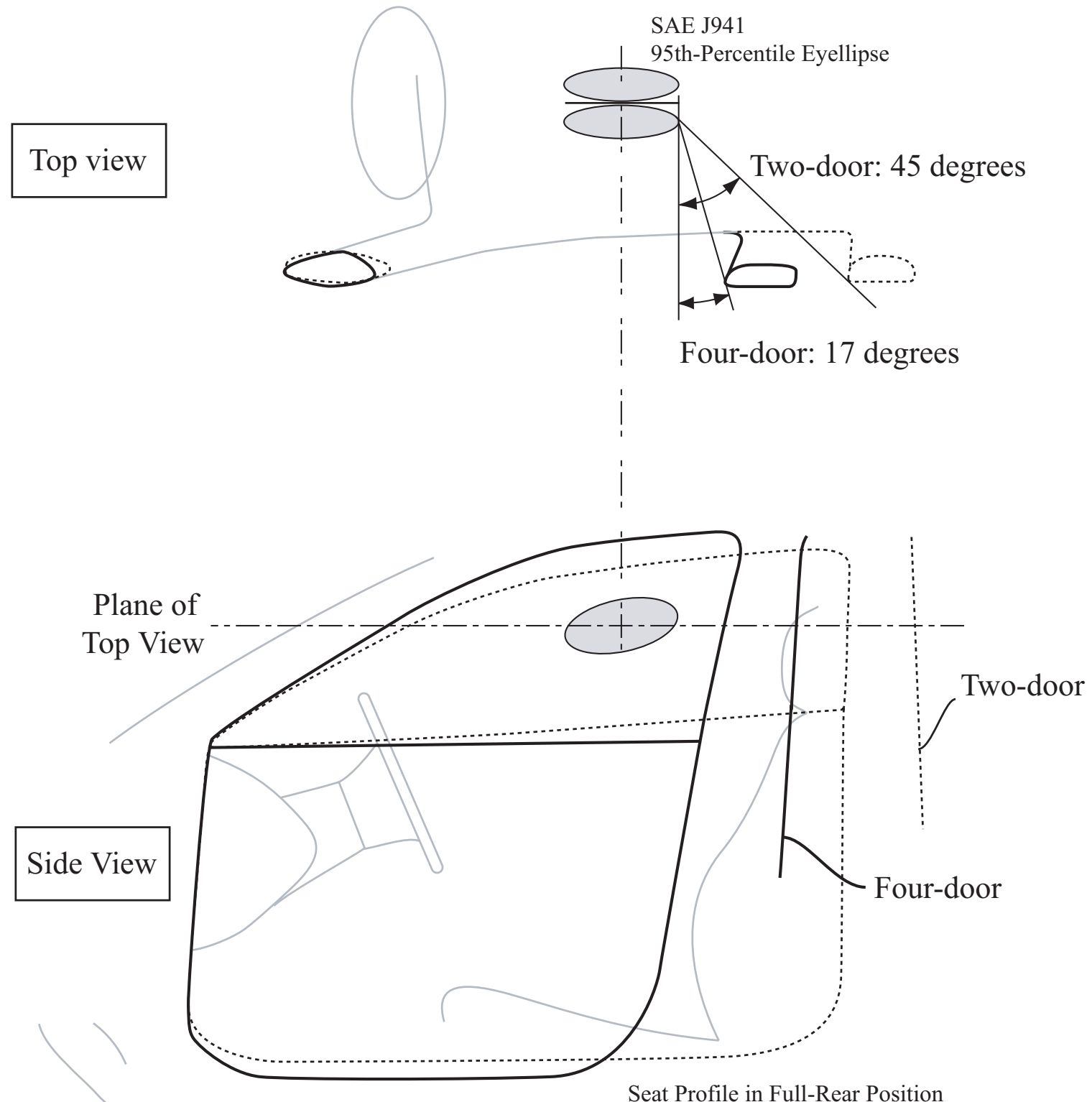

Figure 2. A quantification of the differences in lateral visibility from two-door and fourdoor body styles for the same vehicle model. 


\section{Method}

\section{Vehicles}

The sample consisted of pairs of four-door and two-door body styles for 10 different vehicle models, for a total of 20 different body style/vehicle model combinations. The selected combinations were required to have at least five crashes of each relevant type for inclusion. The 10 different vehicle models selected were as follows (in alphabetical order): BMW 3 Series, Chevrolet Cavalier, Chrysler Sebring, Ford Focus, Honda Accord, Honda Civic, Oldsmobile Alero, Pontiac Grand Am, Toyota Camry/Solara, and Toyota Echo. (One of the two-door models had no B-pillars at all.)

\section{Database}

We used 2000-2003 North Carolina crash data (UNC, 2005) to compile crash frequencies for the selected vehicles. This database includes all reportable North Carolina traffic crashes (fatal, injury, and property damage). The VINDICATOR program (IIHS, 2005) was employed to decode the vehicle identification number (VIN) for each vehicle to select the desired vehicle models, body styles (two-door or four-door), and model years (1995 and newer). Only cases where the model year in the police accident report matched the model year as decoded by VINDICATOR were included.

Crash frequencies were collected for the following crash-related vehicle maneuvers (variable 149): “changing lanes or merging” (vehicle maneuver code: 05), and "going straight ahead" (vehicle maneuver code: 04).

\section{Odds ratio}

The influence of the B-pillar on lane change crashes was evaluated by comparing, for each body style, the likelihood of lane-change crashes in relation to the likelihood of going-straight-ahead crashes. Specifically, for each body style we calculated the ratio of the frequencies of lane-change crashes to going-straight-ahead crashes. In the final step, we compared these two ratios by creating an odds ratio: a ratio of lane-change crashes to going-straight-ahead crashes for four-door models divided by the analogous ratio for twodoor models. 
An odds ratio of 1 would indicate no difference between the two body styles in terms of the relative frequency of lane-change crashes. An odds ratio greater than 1 would indicate an over-involvement of four-door models in lane-change crashes; conversely, an odds ratio of less than 1 would indicate an over-involvement of two-door models in lane-change crashes. 


\section{Results}

Table 1 shows the distribution of crash-related vehicle maneuvers by body style. The odds ratio for the data in Table $1(2,126 / 38,911) /(740 / 15,898)$ is 1.17 , indicating that the odds of four-door models being involved in lane-change crashes are $17 \%$ higher than the corresponding odds for two-door models. The $95 \%$ confidence interval for the obtained odds ratio (1.08 to 1.28) does not include 1, implying that the finding of fourdoor models being over-involved in lane-change crashes is statistically reliable. (The above calculations are for the combined data of all 10 pairs of vehicles. On the individual vehicle level, 9 out of 10 odds ratios are greater than 1.)

Table 1.

Distribution of crash-related vehicle maneuvers by body style.

\begin{tabular}{|c|c|c|}
\hline \multirow{2}{*}{ Vehicle style } & \multicolumn{2}{|c|}{ Crash-related vehicle maneuver } \\
\cline { 2 - 3 } & Lane change & Going straight ahead \\
\hline \hline Two-door & 740 & 15,898 \\
\hline Four-door & 2,126 & 38,911 \\
\hline
\end{tabular}




\section{Discussion}

The results of this study support the hypothesis that lateral visibility out of the vehicle cabin has an effect on safety. Specifically, our data suggest that vehicles with Bpillars located farther forward, and thus nearer the fore-aft position of the driver, tend to be over-involved in lane-change crashes. Future studies should evaluate the sensitivity of this effect to factors such as speed and road type. Of interest would also be the severity of the crashes (fatal, injury, or property damage) that are most affected by this particular obstruction to lateral visibility.

The analysis used two styles of the same vehicle models (two- and four-door body styles, with four-door styles having the B-pillars located farther forward). To account for driver differences between the two vehicle styles and the resulting differences in driving exposure and driving style, crashes in which the vehicles were going straight ahead were used as controls. This particular type of crash was selected because it is unlikely to be affected by lateral visibility. Consequently, the main finding depends on the extent to which the going-straight-ahead crashes, indeed, control for all relevant factors other than lateral visibility.

In terms of a methodological contribution, this study has identified a crash maneuver that is sensitive to a specific obstruction in the driver's direct line of sight (the B-pillar). Furthermore, because there are other potentially relevant obstructions (e.g., Aand C-pillars, roof, and rear deck), this study provides a general approach to quantifying the importance of minimizing a particular visual obstruction. The critical issue in extending the present approach to studying other obstructions is the identification of a crash maneuver (or condition in general) that has a face valid relation to the obstruction in question.

An ideal vehicle would have no obstructions in the driver's line of sight in all relevant directions. However, because of structural-integrity considerations, such an ideal is currently not within reach. Nevertheless, research on sensitivity of crash-

involvement to different visual obstructions would result in quantifying the total magnitude of the problem and, consequently, in setting priorities for minimizing particular visual obstructions. 


\section{References}

IIHS [Insurance Institute for Highway Safety]. 2005. VINDICATOR software. Arlington, VA: Highway Loss Data Institute of the Insurance Institute for Highway Safety.

SAE [Society of Automotive Engineers]. (1995). Devices for use in defining and measuring vehicle seating accommodation (SAE Standard J826). Warrendale, PA: Society of Automotive Engineers.

SAE [Society of Automotive Engineers]. (2002). Motor vehicle drivers' eye locations (SAE Standard J941). Warrendale, PA: Society of Automotive Engineers.

UNC [University of North Carolina]. (2005). North Carolina Crash Data, 2003. Chapel Hill, NC: University of North Carolina Highway Safety Research Center. 

